\title{
Welander's distal myopathy: clinical, neurophysiological and muscle biopsy observations in young and middle aged adults with early symptoms
}

\author{
Kristian Borg, Gabrielle Åhlberg, Jörgen Borg, Lars Edström
}

\begin{abstract}
Nine young or middle aged patients with early symptoms of Welander's distal myopathy were subjected to a detailed neurological examination including quantitative sensory testing, determination of motor and sensory nerve conduction velocity (NCV), sensory nerve action potentials, electromyography (EMG) and muscle biopsy from the tibialis anterior muscle (TA). Slight weakness of the extensors of the fingers and hands was found in all nine patients, and of the dorsiflexors of the feet in seven. All patients had a distal sensory disturbance most prominent for temperature which agrees with earlier observations. EMG changes in TA and extensor digitorum communis (EDC) muscles were of myopathic type. Slight abnormalities compatible with either myopathy or early neuropathy were found in one muscle biopsy. These findings indicate that a neurogenic lesion affecting at least the peripheral sensory system is present at an early stage of Welander's distal myopathy and that the neurogenic lesion might precede the myopathic changes.
\end{abstract}

In 1951 Welander ${ }^{1}$ described a distal myopathy in 249 cases from 72 pedigrees with an autosomal dominant mode of inheritance. Onset before the age of 30 was exceedingly rare and an onset before the age of 40 was as uncommon. However, in the study by Welander ${ }^{1}$ three affected individuals had an early onset of symptoms, at the ages of 20,27 and 29 , respectively.

The histopathological changes in affected muscles were described by Welander ${ }^{1}$ as being typical of a primary myopathy and similar to other muscular dystrophies. Data from electromyographic ${ }^{2-5}$ and muscle fibre histochemical and ultrastructural studies, ${ }^{6-8}$ however, have suggested a neurogenic component which might be primary, secondary or parallel.

Sensory abnormalities were originally described by Welander ${ }^{1}$ but were ascribed to coincidental disorders and ageing. Recently, a sensory nerve dysfunction in both myelinated and unmyelinated nerve fibres ${ }^{89}$ and a loss of small diameter myelinated nerve fibres in the sural nerve ${ }^{8}$ have been described in Welander's distal myopathy giving a further support for a neurogenic component.
The aim of this study was to characterise the early stage of Welander's distal myopathy with reference to clinical, electrophysiological and muscle biopsy findings and to clarify whether the neurogenic component is an early and primary, or late and possibly secondary phenomenon.

\section{Materials and methods}

Nine patients, five women and four men, 23-47 years of age (median 31 years) were selected from a group of 20 young or middleaged relatives of patients who clinically and neurophysiologically fulfilled the criteria for a hereditary distal myopathy as described by Welander. ${ }^{1}$ The selection was made on the basis of abnormalities found on routine neurological examination. The nine patients were members of six families and in each of these families there was at least one member who was originally diagnosed by Welander.

A detailed neurological examination was carried out. Sensibility screening was performed with cotton wool, pinprick, graphaesthesia, and warm and cold metallic rollers of $40^{\circ} \mathrm{C}$ and $20^{\circ} \mathrm{C}$, respectively.

Thermal thresholds and thresholds for thermal pain were determined in the thenar region of the hand and on the lateral aspect of the foot according to Fruhstorfer et al 1976. ${ }^{10}$ The warm and cold thresholds were defined as the temperatures at which the patients perceived warm and cold, respectively, and the warm-cold difference limen as the difference between those two values. The thermal pain threshold was defined as the temperature at which the stimulus was perceived as painful.

Vibration thresholds were determined over the carpal, tibial and tarsal bones with an electromagnetic vibrator using Goldberg and Lindblom's method. ${ }^{11}$ An average was calculated from at least three determinations at each site and the threshold was defined as the average of the amplitude at which vibration was first perceived and the amplitude at which the sensation disappeared on subsequent stimulus reduction. Data from determinations of thermal and vibratory thresholds were compared with data from reference materials of healthy, normal subjects. ${ }^{11}$ Deviations of more than $\pm 2 \mathrm{SD}$ were considered as abnormal.

Determination of sensory and motor nerve conduction velocities (NCV) were performed in peroneal, median and sural nerves using standard techniques. Temperature corrected 
conduction velocity values were compared with age matched control data.

Electromyography (EMG) was performed in the tibialis anterior muscle (TA) and the extensor digitorum communis (EDC) muscle. Concentric needle electrodes (Medelec 22585) were used for routine examinations and for computerised analysis of interference pattern and motor unit potentials according to Stålberg et $a l,{ }^{12}$ (using Intersoft programs TA and MUP, version 3 1988).

Single fibre needle electrodes (Medelec 16829) were used to determine fibre density and jitter using the method of Stålberg and Trontelj. ${ }^{13}$

EMG recordings were displayed either on a Medelec oscilloscope (MS92B no 67127) connected to a microcomputer (Victor VPCII $611112780 \mathrm{C}$ ) or on a Medelec oscilloscope (MS6 no 4329) with simultaneous printout on Kodak Linagraph Direct print paper for manual measurement of motor unit potential parameters. For the motor unit potential analysis the sweep speed was $1 \mathrm{~mm} / \mathrm{ms}$ using both the computerised and manual method, while the band width was $2 \mathrm{~Hz}-10 \mathrm{kHz}$ or $1 \cdot 6$ $16 \mathrm{KHz}$, respectively, depending on different equipment properties.

\section{Muscle biopsy procedure}

Biopsies were performed in the TA by the percutaneous conchotome method originally described by Radner ${ }^{14}$ and slightly modified by Lindholm. ${ }^{15}$

The biopsy material intended for histochemistry and light microscopical morphology was quickly frozen in Freon 13 kept at its melting point $\left(-190^{\circ} \mathrm{C}\right)$ in liquid nitrogen and kept in a refrigerator at $-75^{\circ} \mathrm{C}$ until further processed. Sections of $10-15 \mu \mathrm{m}$ were cut in a cryostat operating at $-25^{\circ} \mathrm{C}$. The biopsy sections were stained with haematoxylin-eosin, modified trichrome according to Engel and Cunningham, ${ }^{16}$ myosin adenosine triphosphatase (ATPase) according to the original method of Padykula and Her$\operatorname{man}^{17}$ and the modifications by Brooke and Kaiser $^{18}$ and NADH-TR. ${ }^{19}$

The muscle fibre nomenclature was based on the ATPase stainability according to Brooke and Kaiser. ${ }^{18}$ Thus fibres with a high content of acid stable ATPase and low content of alkali stable ATPase were termed "type I" while fibres with the opposite staining pattern were termed "type II". All fibres on a muscle biopsy cross-section were classified and the total number of fibres of each type estimated. Cross-sectional areas of individual muscle fibres from each biopsy were measured by a semi-automatic technique applied on black and white photomicrographs of muscle cross-sections (MOP Videoplan, Kontron, Munich). Muscle fibre data were compared with data from normal controls presented earlier. ${ }^{20}$ Deviations of more than $\pm 2 \mathrm{SD}$ from those values were considered abnormal.

\section{Results}

Eight of nine patients admitted some stiffness in the fingers and clumsiness especially at cold exposure. A common complaint was a difficulty in using the opposed thumb and index finger grip against resistance, for example, when turning the key of a stiff lock. Two patients complained of slight walking problems and one was examined at the age of 16 and was already then unable to walk on her heels.

The present clinical neurological examination revealed slight extensor weakness of the hands and fingers in all patients. Seven of the patients had moderate dorsiflexor weakness of the feet and had difficulty in walking on their heels. No proximal weakness and no obvious muscle atrophy were found in any of the patients. One patient had bilateral absence of the achilles tendon reflexes. The other tendon reflexes in this patient and all tendon reflexes in the other eight patients were normal.

For results of the sensibility screening and quantitative sensory testing see fig 1 . All patients had hypoaesthesia for thermal sensibility distally in the lower extremities on screening. In addition, three of the patients had impaired sensibility for touch and six had impaired sensibility for pain (one had hyperalgesia) in the feet. Sensibility impairment for temperature in the hands was found in seven patients, for touch in three patients and for pain in these three and another two. Graphaesthesia was normal in all patients.

Six of the patients had abnormal thermal thresholds in the feet. Four of these patients had abnormal thresholds in the hands as well. Four patients had a paradoxical warm sensation on cold stimulation of the feet (fig 2). Three patients had abnormal thermal pain thresholds in both the hands and feet.

All patients had normal vibratory thresholds over the carpal and tarsal bones but three had abnormal thresholds over the tibial bone.

Sensory and motor NCV were within normal range as were the sensory nerve action potential amplitudes of the median, peroneal and sural nerves.

All patients were subjected to routine EMG analysis. EMG recordings in the TA and EDC included quantitative EMG in seven patients.

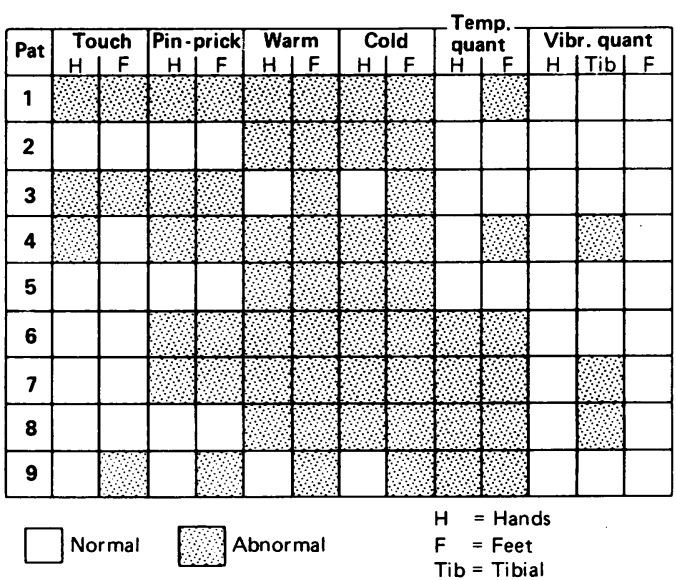

Figure 1 Results of the sensibility screening and quantitative sensory testing (Temp quant = thermal and thermal pain thresholds and Vibr quant = vibratory thresholds) of the extremities in the nine patients with Welander's distal myopathy. 


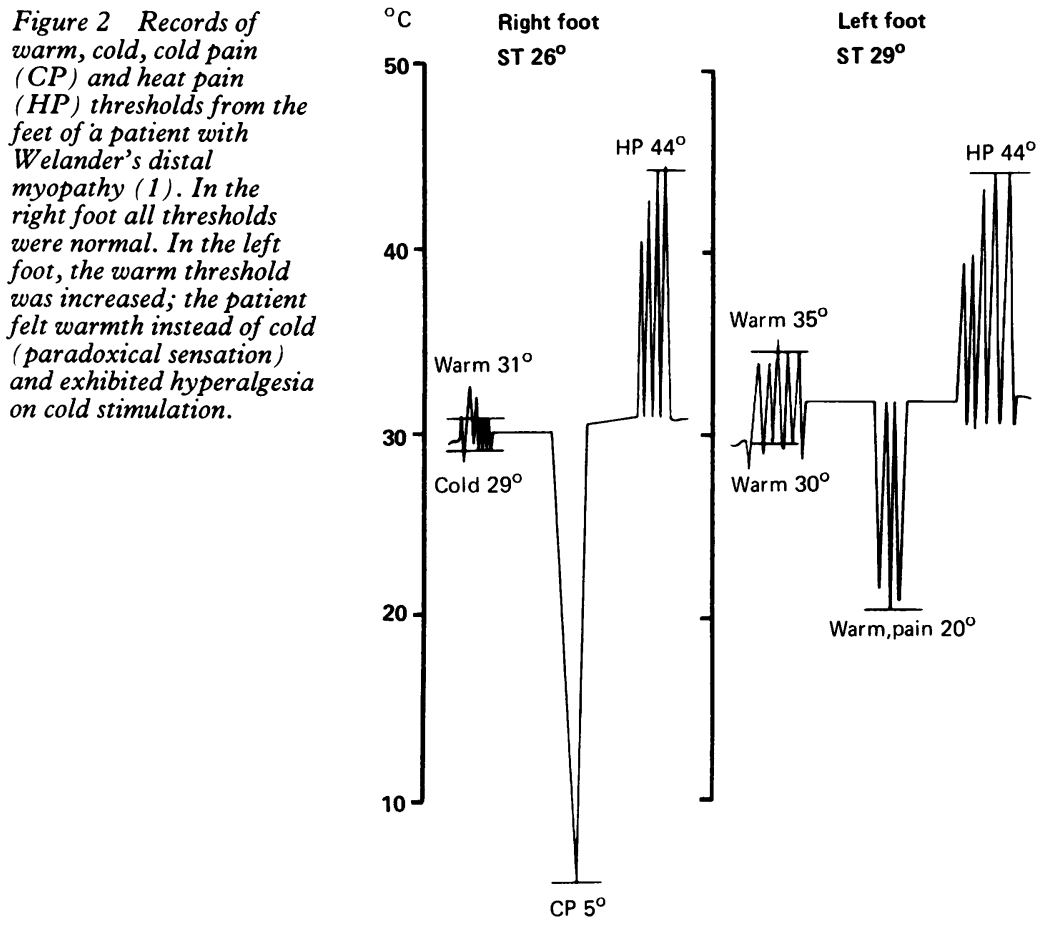

There was no pathological spontaneous activity. A complete interference pattern was achieved at maximal voluntary effort in all muscles. Automatic analysis of the EMG interference pattern ${ }^{12}$ was abnormal according to the defined criteria (two or more data outside given limits) in 2/14 investigated muscles (patient 1 and 7 in the table). In the other 12/14 muscles data were within normal limits.

Motor unit potentials (MUPs) were analysed manually in $3 / 7$ patients (1-3) and computerised in all seven patients. At both procedures 20 or more motor units were analysed in each muscle. Computerised data are summarised in the table.

MUP amplitudes were within the low range at manual and computerised analysis. For 202 TA motor units the amplitudes were $<1 \mathrm{mV}$ in $85 \%, 1-2 \mathrm{mV}$ in $12 \%$ and $2-3 \mathrm{mV}$ in $3 \%$. Corresponding figures from 188 EDC motor units were $93 \%, 5 \%$ and $3 \%$.

MUP durations were ordinary or short. At manual analysis the mean values were $9 \cdot 4$ $10.5 \mathrm{~ms}$ in three TA muscles, which was at or below -2 SD when compared with corresponding data from healthy subjects. ${ }^{21}$ At computerised analysis the mean values were $7 \cdot 49$ $13.57 \mathrm{~ms}$ in TA and 7.49-10.92 in EDC as shown in the table. These data (table) were

Mean values for motor unit potential amplitude and duration at computerised analysis of 20 or more motor unit potentials (MUPs) of TA and EDC in seven of the patients with Welander's distal myopathy. Mean values of MUP duration from Ludin ${ }^{21}$ are shown in parenthesis. The patient numbers correspond to those of fig 1 ( $f=$ female, $m=$ male). Patients 4 and 9 in fig 1 are not included in the quantitative EMG study

\begin{tabular}{|c|c|c|c|c|}
\hline \multirow[b]{2}{*}{ Patients } & \multicolumn{2}{|c|}{ MUP amplitude $(m V)$} & \multicolumn{2}{|c|}{ MUP duration (ms) } \\
\hline & $T A$ & $E D C$ & $T A$ & $E D C$ \\
\hline $\begin{array}{ll}1 & (\mathrm{f}) \\
2 & (\mathrm{f}) \\
3 & (\mathrm{f}) \\
4 & (\mathrm{~m}) \\
6 & (\mathrm{~m}) \\
7 & (\mathrm{~m}) \\
8 & (\mathrm{f})\end{array}$ & $\begin{array}{l}0.37 \\
0.35 \\
0.49 \\
0.92 \\
0.71 \\
0.61 \\
0.70\end{array}$ & $\begin{array}{l}0.82 \\
0.64 \\
0.33 \\
0.76 \\
0.36 \\
0.47 \\
0.50\end{array}$ & $\begin{array}{r}8 \cdot 18(13 \cdot 1) \\
8.81(13 \cdot 1) \\
8.39(13 \cdot 1) \\
10 \cdot 72(12 \cdot 7) \\
10 \cdot 29(13 \cdot 8) \\
7.49(13 \cdot 8) \\
13.57(14 \cdot 0)\end{array}$ & $\begin{aligned} & 10.92(9.8) \\
& 9.43(9.8) \\
& 7.61(9.8) \\
& 9.30(9.5) \\
& 8.19(10.3) \\
& 7.49(10.3) \\
& 9.58(10.5)\end{aligned}$ \\
\hline
\end{tabular}

more than 2 SD below the published normal values $^{21}$ in the TA of four patients $(1,2,3$, and 7) and in two of these patients ( 3 and 7 ) also in EDC. However, statistical analysis of the computerised data was not possible since normal values are so far lacking.

MUP polyphasia was prominent in four patients (1, 2, 3, and 7). Two of these ( 3 and 7 ) were also abnormal using the automatic analysis of interference pattern described earlier. Manual measurement showed abnormal polyphasia with four or more base line crossings $^{21}$ in $20-50 \%$ of the motor units of three TAs. Computerised analysis showed two or more highly polyphasic potentials (five or more base line crossings) in the TA in all four patients and in EDC in one of them (2).

Fibre density was within normal limits $( \pm 2$ $\mathrm{SD}$ ) in TA and EDC of all patients investigated. Abnormal jitter or blockings were not observed. ${ }^{13}$

Based upon the data presented, EMG was considered abnormal in the four patients $(1,2$, 3 , and 7) with MUPs exhibiting low amplitude, short duration and polyphasia in TA. According to conventional criteria these changes were of "myopathic" type. Similar but less pronounced changes were seen in the other patients.

The TA muscle biopsies from eight patients did not show any morphological abnormalities. There was a normal range of fibre crosssectional areas and no atrophic fibres were found. In the last patient (7) there was a small number of atrophic, angulated fibres some with rimmed vacuoles but there were no structural abnormalities in the normal sized fibres (fig 3). Muscle fibre differentiation was present in all biopsies and permitted an identification of type I and II muscle fibres according to conventional criteria of stainability for ATPase. The type I muscle fibre percentage was within the normal range as compared with data from healthy individuals. ${ }^{20}$

\section{Discussion}

Our young or middle aged patients were members of families in which Welander's distal myopathy occurs with an autosomal dominant inheritance. All patients had slight clinical signs including weakness of extensors of the fingers and hands and in most cases also of the dorsiflexors of the feet. These findings conform with the typical clinical picture described by Welander. ${ }^{1}$ Thus it is obvious that these patients had early symptoms of Welander's distal myopathy, that the disease might have an early onset and that a sensory dysfunction seems to be an early sign of the disorder. There is a prerequisite for a significant patient delay because of the mild symptoms and slow progression rate of symptoms.

Impaired sensibility, most pronounced for thermal stimulation, and a corresponding loss of small diameter myelinated nerve fibres in the sural nerve was recently found in typical cases of long standing Welander's distal myopathy. ${ }^{89}$ The sensory dysfunction observed in all our patients agrees with this finding and strongly 
Figure 3 .

Photomicrographs of a $T A$ muscle biopsy from patient 7. Cryostat cross-sections are stained for $\mathrm{Htx}$-eosin ( $a$ and d), myosin ATPase after acid preincubation at pH $4 \cdot 6$ (b) and NADH-TR (c). Fig $a-c$ are serial sections and a cross facilitates the comparison. In $d$ is shown an enlargement of a detail from $a$. $A$ bar in all sections indicate $50 \mu \mathrm{m}$. Note the scattered atrophic, elongated fibres, some of which are strongly stained for $N A D H-T R$. Arrows in a and dindicate rimmed vacuoles. Fibres of type I, IIA and IIB are observed in $b$ and there is no convincing type grouping but rather a type I fibre dominance.
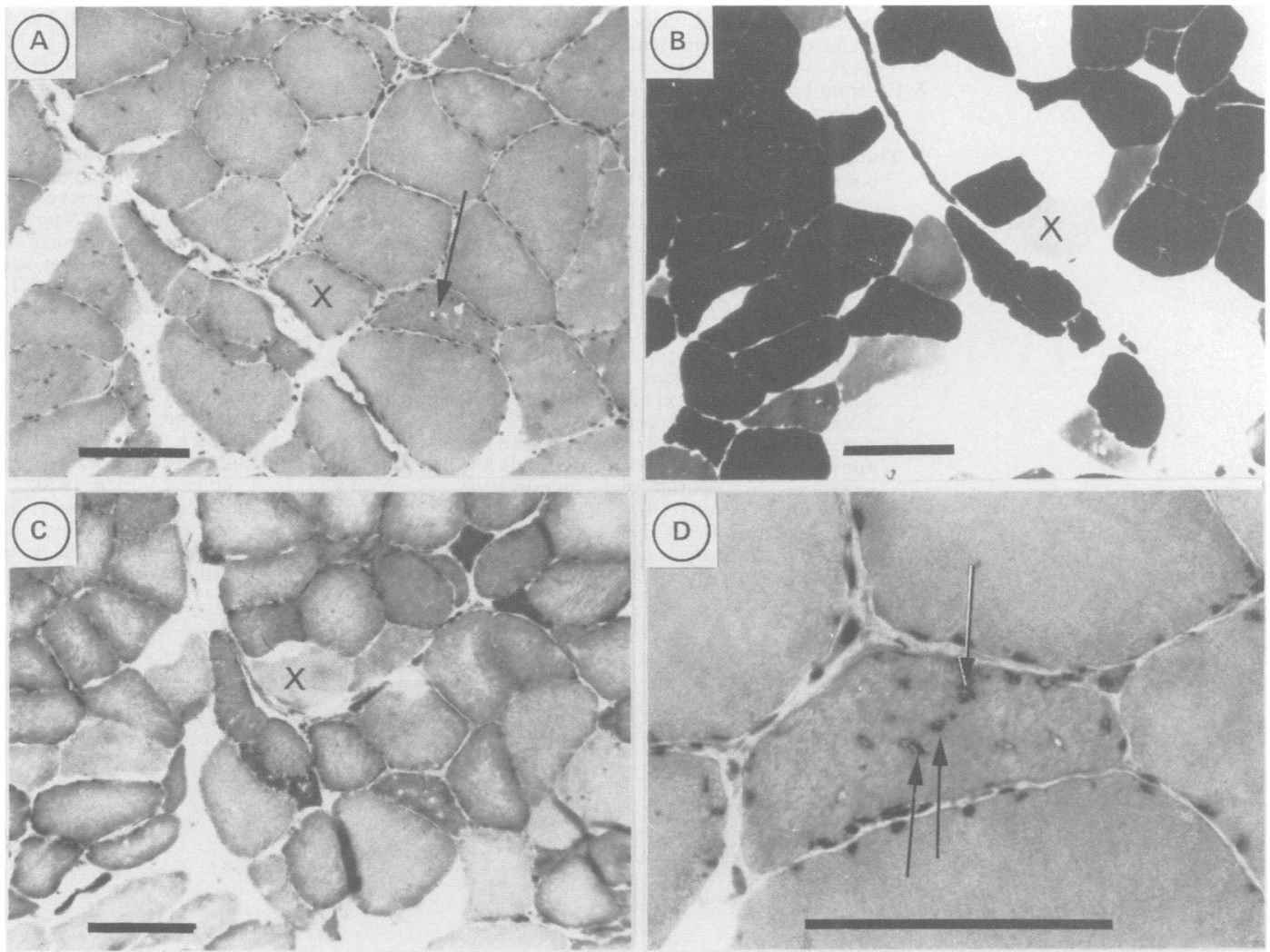

suggests that this is a typical early phenomenon in Welander's distal myopathy. Three of the patients had normal thermal thresholds on quantitative sensory testing although their thermal sensibility was abnormal on screening. This phenomenon was recognised in our previous study ${ }^{9}$ and is most probably explained by hypoaesthesia confined to the suprathreshold range as suggested by Lindblom and Ochoa. ${ }^{22}$

To characterise and quantitate early EMG changes in Welander's disease the conventional EMG was combined with computerised methods for interference pattern analysis and motor unit potential analysis. The interference pattern analysis showed statistically significant "myopathic" pattern in two examined muscles but no muscles with a "neurogenic" pattern. Motor unit fibre density was normal in all muscles. Motor unit potential durations were short when compared with published normal values. ${ }^{21}$ There was an increased incidence of polyphasic potentials at both manual ${ }^{21}$ and computerised analysis. It is concluded that when EMG changes occur in cases with early symptoms of Welander's disease they are of "myopathic" type. These changes are then compatible with a primary myopathic process but might also be compatible with terminal axonal twig neuropathy in combination with reduced reinnervation capacity. The lack of spontaneous activity in resting muscle, however, favours a myopathy.

In previous histopathological studies of TA muscle in patients with long-standing Welander's distal myopathy, extensive light microscopical and ultrastructural changes were found. ${ }^{6-8} \mathrm{~A}$ loss of muscle fibre differentiation was typical in advanced cases. ${ }^{6}$ In our early cases of Welander's distal myopathy no mor- phological muscle fibre abnormalities were found in eight of nine cases. When considering the lack of significant histopathological changes in spite of electromyographic abnormalities in the corresponding patients it should be remembered that the muscle biopsy represents a smaller sampling area than the EMG which picks up signals from different needle positions. Another explanation for this finding could be that the muscle disorder primarily affects the muscle fibre membrane and not the contractile apparatus. Yet another explanation is the possibility of a terminal twig neuropathy suggested by Stålberg and Ekstedt. ${ }^{4}$

The sensory dysfunction and the previously reported loss of small diameter myelinated nerve fibres in the sural nerve in patients with Welander's distal myopathy ${ }^{89}$ conclusively demonstrates that a peripheral nerve lesion precedes or coincides with the muscle affection. Thus the mutant gene might be expressed as an abnormality both in nerve and muscle tissue.

This study was supported by grants from the Swedish Medical Research Council (3875), the Swedish MS foundation, the Swedish Society for Traffic and Polio Disabled, the Swedish Society of Medicine, the Norrbacka-Eugenia foundation and from the Karolinska Institute. We are indebted to Ms Birgitta Hedberg and Ms Birgitta Lindegren for excellent technical assistance.

1 Welander L. Myopathia distalis tarda hereditaria. Acta Med Scand 1951;141:1-124.

2 Kugelberg E. Electromyogram in muscular disorders. $J$ Neurol Neurosurg Psychiatry 1947;10:122-33.

3 Kugelberg E, Petersén I. "Insertion activity" in electromyography with notes on denervated muscle response to constant current. J Neurol Neurosurg Psychiatry 1947;12: 268-73.

4 Stålberg E, Ekstedt J. Signs of neuropathy in distal hereditary myopathy (Welander). Electroenceph Clin Neurophysiol 1969;26:343.

5 Borg J, Grimby L, Hannerz J. Motor neuron firing rates 
axonal conduction velocity, and muscle fiber histochemistry in neuromuscular diseases. Muscle Nerve 1979; 2:423-30.

6 Edström L. Histochemical and histopathological changes in skeletal muscles in late-onset hereditary myopathy (Welander). J Neurol Sci 1975;26:147-57.

7 Thornell L-E, Edström L, Billeter R, Butler-Browne GS, Kjörell U, Whalen RG. Muscle fibre type composition in distal myopathy (Welander). An analysis with enzymeand immuno-histochemical, gelelectrophoretic and

8 ultrastructural techniques. J Neurol Sci 1984;65:269-92. Borg K, Solders G, Borg J, Edström L, Kristensson K.
Neurogenic involvement in distal myopathy (Welander). J Neurol Sci 1989;91:53-70.

9 Borg K, Borg J, Lindblom U. Sensory involvement in distal myopathy (Welander). J Neurol Sci 1987;80:323-32.

10 Fruhstorfer H, Lindblom U, Schmidt WG. Method for quantitative estimation of thermal thresholds in patients. $J$ Neurol Neurosurg Psychiatry 1976;39:1071-5.

11 Goldberg JM, Lindblom U. Standardized method of determining vibratory perception thresholds for diagnosis and Neurosurg Psychiatry 1979;42:793-803.

12 Stålberg E, Chu J, Bril V, Nandekar S, Stàlberg S, Eriksson $M$. Automatic analysis of the EMG interference pattern. EEG Clin Neurophysiol 1983;56:672-81.
13 Stålberg E, Trontelj JV. Single fibre electromyography. Old Woking, Surrey: The Mirvalle Press, 1979.

14 Radner S. Knappnålsteknik för iterativ muskelbiopsi. Trans Swedish Soc Med Sci 1962;XIX:94.

15 Lindholm $T$. The influence of uraemia and electrolyte disturbances on muscle action potentials and motor nerve conduction in man. Acta Med Scand Suppl 1968:491.

16 Engel WK, Cunningham GC. Rapid examination of muscle tissue-An improved trichrome method for fresh-frozen biopsy sections. Neurology (Minneap) 1963;13:919.

17 Padykula HA, Herman E. The specificity of the histochemical method of adenosine triphosphatase. J Histochemical method of adenosine

18 Brooke MH, Kaiser KK. Muscle fiber types-How many and what kind? Arch Neurol (Chic) 1970;23:369-70.

19 Dubowitz V, Brooke MH. Muscle biopsy-A modern approach. Philadelphia: W B Saunders, 1973:34-102.

20 Jakobsson F, Borg K, Edström L, Grimby L. Use of motor units in relation to muscle fiber type and size in man. Muscle Nerve 1988;11:1211-8.

21 Ludin HP. Electromyography in practice. Stuttgart: George Thieme Verlag, 1980.

22 Lindblom U, Ochoa J. Somatosensory function and dysfunction. In: Ashbury AK, McKhann G, McDonald WI, eds. Diseases of the nervous system. Clinical neurobiology. Philadelphia: W B Saunders, 1986:727-35. 